\title{
ON THE ORDER OF ARC-STABILIZERS IN ARC-TRANSITIVE GRAPHS
}

\author{
GABRIEL VERRET
}

(Received 18 March 2009)

\begin{abstract}
Let $p$ be a prime. We say that a transitive action of a group $L$ on a set $\Omega$ is $p$-sub-regular if there exist $x, y \in \Omega$ such that $\left\langle L_{x}, L_{y}\right\rangle=L$ and $L_{x}^{Y} \cong \mathbb{Z}_{p}$, where $Y=y^{L_{x}}$ is the orbit of $y$ under $L_{x}$. Our main result is that if $\Gamma$ is a $G$-arc-transitive graph and the permutation group induced by the action of $G_{v}$ on $\Gamma(v)$ is $p$-sub-regular, then the order of a $G$-arc-stabilizer is equal to $p^{s-1}$ where $s \leq 7, s \neq 6$, and moreover, if $p=2$, then $s \leq 5$. This generalizes a classical result of Tutte on cubic arc-transitive graphs as well as some more recent results. We also give a characterization of $p$-sub-regular actions in terms of arc-regular actions on digraphs and discuss some interesting examples of small degree.
\end{abstract}

2000 Mathematics subject classification: primary 05C25; secondary 20B25.

Keywords and phrases: arc-transitive graph, arc-stabilizer.

\section{Introduction}

Throughout this paper, all graphs considered will be finite, simple and connected. Let $\Gamma$ be such a graph and let $G \leq \operatorname{Aut}(\Gamma)$. Then $\Gamma$ is said to be $G$-arc-transitive $(G$ arc-regular) if $G$ acts transitively (regularly) on arcs of $\Gamma$. The study of arc-transitive graphs was initiated by Tutte [16]. His classical result is that if $\Gamma$ is a cubic $G$-arctransitive graph, then $G$ acts regularly on $s$-arcs for some $s \leq 5$ and the order of the arc-stabilizer $G_{u v}$ is $2^{s-1}$.

Since then, arc-transitive graphs have been widely studied. It is well known that the order of an arc-stabilizer is unbounded for arc-transitive graphs in general. Nevertheless, some results can be obtained by considering the local action of an arctransitive group, which we now define.

If $G$ is a group acting on a set and $\Theta$ is a union of orbits under this action, then $G^{\Theta}$ will denote the permutation group induced by the action of $G$ on $\Theta$. Let $\Gamma$ be a $G$ arc-transitive graph, let $v$ be a vertex of $\Gamma$ and let $\Gamma(v)$ denote the neighborhood of $v$ in $\Gamma$. We call $G_{v}^{\Gamma(v)}$ the local action of $(\Gamma, G)$. Moreover, if $P$ is a permutation group property and $G_{v}^{\Gamma(v)}$ has property $P$, then we say that $(\Gamma, G)$ is a locally $P$ pair.

(C) 2009 Australian Mathematical Publishing Association Inc. 0004-9727/2009 \$16.00 
Let $k$ denote the valency of $\Gamma$. Note that, since $\Gamma$ is $G$-arc-transitive, $G_{v}^{\Gamma(v)}$ is a transitive subgroup of the symmetric group $S_{k}$ and, up to equivalence, does not depend on the choice of $v$. Sami [10] studied locally $\mathrm{D}_{k}$ pairs $(\Gamma, G)$ where $\mathrm{D}_{k}$ denotes the dihedral group of order $2 k$ acting naturally on a set of cardinality $k$. In particular, he showed that, if $k$ is odd, the order of a $G$-arc-stabilizer divides $2^{4}$, generalizing Tutte's result. In this paper, we further extend this result by obtaining similar bounds for a wider class of local actions, which we now define.

Definition 1.1. Let $p$ be a prime. We say that a transitive action of a group $L$ on a set $\Omega$ is $p$-sub-regular if there exist $x, y \in \Omega$ such that $\left\langle L_{x}, L_{y}\right\rangle=L$ and $L_{x}^{Y} \cong \mathbb{Z}_{p}$, where $Y=y^{L_{x}}$ is the orbit of $y$ under $L_{x}$.

One can easily see that the natural action of $\mathrm{D}_{k}$ is 2-sub-regular for every odd integer $k$. A characterization of $p$-sub-regular actions in terms of half-arc-transitive actions on graphs will be given in Section 3, and several families of $p$-sub-regular actions will be described. With this definition, we can now state our main result.

THEOREM 1.2. Let $(\Gamma, G)$ be a locally $p$-sub-regular pair and let $u v$ be an arc of $\Gamma$. Then the order of the arc-stabilizer $G_{u v}$ is equal to $p^{s-1}$ where $s \leq 7, s \neq 6$. Moreover, if $p=2$, then $s \leq 5$.

Since dihedral groups of odd degree are 2-sub-regular, this is a generalization of Sami's (and hence Tutte's) result. These results can be viewed as part of a more general problem. Call a transitive permutation groups $L$ restrictive if there exists a constant $c(L)$ such that, for any locally $L$ pair $(\Gamma, G)$, the inequality $\left|G_{u v}\right| \leq c(L)$ holds.

PROBLEM 1.3. Characterize restrictive permutation groups.

A lot of work has been done on this problem. A trivial observation is that regular groups are restrictive. The previously mentioned results of Tutte and Sami show that odd dihedral groups are restrictive. Gardiner [4, 5] showed that doubly primitive groups are restrictive. In the same vein, Weiss [18] conjectured that primitive groups are restrictive. The conjecture was recently verified in the 2-transitive case [12-15] but the general conjecture is still quite open. See $[2,7]$ for some recent results.

Besides purely theoretical benefits, obtaining such upper bounds on the order of arc-stabilizers for graphs of a certain family also yields an efficient way to enumerate the graphs of this family up to a certain order. See [1, 3, 9] for examples.

Apart from $\mathrm{D}_{4}$, all transitive actions of degree at most 5 are either regular, 2transitive or $p$-sub-regular, for some $p$. It is well known that the order of arc-stabilizers of locally $\mathrm{D}_{4}$ pairs is unbounded. Hence, with respect to Problem 1.3, the smallest open degree is 6 . As a starting point to solving Problem 1.3, it would therefore be interesting to classify transitive groups of degree 6 according to the existence of such a bounding constant.

Section 2 will be devoted to the proof of Theorem 1.2. In Section 3, we give a characterization of $p$-sub-regular actions. We also give a list of $p$-sub-regular actions that is exhaustive up to degree 26. 


\section{Proof of Theorem 1.2}

Our group theory notation is rather standard and follows Wielandt [19]. If $x, g$ are elements of a group $G$, we will denote by $x^{g}=g^{-1} x g$ the conjugate of $x$ by $g$. Similarly, if $X \subseteq G$, then $X^{g}$ will denote $\left\{x^{g} \mid x \in X\right\}$. Finally, if $G$ is a group acting on a set $\Omega$ and $x \in \Omega$, then $x^{G}$ will denote the orbit of $x$ under $G$.

Our notation and terminology with respect to graphs is also fairly standard. Even though our main interest lies in simple graphs, it will be convenient for us to view graphs in a slightly more general context of digraphs. A digraph $\Gamma=(V, A)$ consists of a finite set of vertices $V$ and a set of $\operatorname{arcs} A \subseteq(V \times V) \backslash\{(v, v) \mid v \in V\}$. For an arc $(u, v)$ of a digraph $\Gamma$, we say that $u$ and $v$ are the vertices of $(u, v)$. Also, we say that $v$ is an out-neighbor of $u$ and that $u$ is an in-neighbor of $v$. The symbols $\Gamma^{+}(v), \Gamma^{-}(v)$ and $\Gamma(v)$ denote the set of out-neighbors of $v$, the set of in-neighbors of $v$ and the union $\Gamma^{+}(v) \cup \Gamma^{-}(v)$, respectively. We call $\left|\Gamma^{+}(v)\right|$ and $\left|\Gamma^{-}(v)\right|$ the out-degree and the in-degree of $v$, respectively. If the out-degree (in-degree) is constant for all $v \in V$, we will call it the out-valency (in-valency) of $\Gamma$. If the in-valency and the out-valency are equal, we call it simply the valency.

If for all $u, v \in V$, we find that $(u, v) \in A$ whenever $(v, u) \in A$, we say that $\Gamma$ is a graph. An edge of a graph is an unordered pair $\{u, v\}$ (also denoted by $u v$ ) such that $(u, v)$ is an arc of the graph.

An $n$-arc $U=\left(u_{0}, \ldots, u_{n}\right)$ is a sequence of $n+1$ vertices of $\Gamma$ such that $\left(u_{i}, u_{i+1}\right) \in A$ for $0 \leq i \leq n-1$ and $u_{i} \neq u_{i+2}$ for $0 \leq i \leq n-2$. We will call $u_{0}$ the start-vertex of $U$ and $u_{n}$ the end-vertex of $U$. Similarly, if $n \geq 1$, then $\left(u_{0}, u_{1}\right)$ will be called the starting arc of $U$ and $\left(u_{n-1}, u_{n}\right)$ will be called the ending arc of $U$. A subsequence of $U$ of the form $\left(u_{i}, u_{i+1}, \ldots, u_{j-1}, u_{j}\right)$ for some $0 \leq i \leq j \leq n$ is called a $\operatorname{sub}-(j-i)$-arc of $U$. An $n$-arc of the form $\left(u_{1}, \ldots, u_{n}, u\right)$ will be called a successor of $U$. Note that the graph $\Gamma$ is connected if and only if, for any pair of vertices $x, y \in V$, there is an $n$-arc (for some $n$ ) with start-vertex $x$ and end-vertex $y$.

We now introduce the notion of nice arcs.

Definition 2.1. Let $\Gamma$ be a graph, let $G \leq \operatorname{Aut}(\Gamma)$, let $(x, y, z)$ be a 2 -arc of $\Gamma$ and let $n \geq 2$ be an integer. An $n$-arc of $\Gamma$ will be called nice (with respect to $(x, y, z)$ and $G)$ if each of its sub-2-arcs is in the orbit of $(x, z, y)$ under $G$. A 1-arc of $\Gamma$ is called nice if it belongs in the $G$-orbit of the arc $(x, y)$.

We will simply call an $n$-arc nice and omit the mention of $(x, y, z)$ and $G$ when ambiguity is unlikely. Note that every 1 -arc is nice if and only if $G$ is arc-transitive. Similarly, every $n$-arc of $\Gamma$ with $n \geq 2$ is nice if and only if $G$ acts transitively on 2-arcs of $\Gamma$. The 2-arc-transitive graphs have been extensively studied and, as we will see, the notion of nice $n$-arcs allows us to apply some of the techniques to the 1 -arc-transitive case.

The following basic result will be used a few times. The proof is a simple exercise. 
LEMMA 2.2. Let $G$ be a finite group acting transitively on a set $\Omega$ and let $R$ be $a$ transitive nonempty relation on $\Omega$. If $R$ is preserved by $G$, then it is an equivalence relation.

Our next aim is to give sufficient conditions for $G$ to act regularly on nice $s$-arcs for some $s \geq 2$.

LEMMA 2.3. Let $p$ be a prime, let $(\Gamma, G)$ be a locally $p$-sub-regular pair, let $z$ be a vertex of $\Gamma$ and let $L=G_{z}^{\Gamma(z)}$. Let $x, y \in \Gamma(z)$ such that $L=\left\langle L_{x}, L_{y}\right\rangle$ and $L_{x}^{Y} \cong \mathbb{Z}_{p}$, where $Y=y^{L_{x}}$. Let s be the largest integer such that $G$ acts transitively on $s$-arcs of $\Gamma$ that are nice with respect to $(x, z, y)$ and $G$. Let $\left(v_{0}, \ldots, v_{s}\right)$ be a nice s-arc of $\Gamma$ and let $G_{i}$ be the pointwise stabilizer of $\left(v_{0}, \ldots, v_{s-i}\right)$. Then, for $0 \leq i \leq s-1$, it follows that $\left|G_{i}\right|=p^{i}$. In particular, $\left|G_{0}\right|=1$ and $G$ acts regularly on nice $s$-arcs.

PROOF. Note that $\left|y^{L_{x}}\right|=p$ implies that a nice $t$-arc has exactly $p$ nice successors. For $1 \leq i \leq s-1$, it follows that $\left|\left(v_{s-i+1}\right)^{G_{i}}\right|=\left|G_{i}: G_{i-1}\right|=p$ and, by induction, that $\left|G_{i}\right|=p^{i}\left|G_{0}\right|$. It remains only to prove that $\left|G_{0}\right|=1$.

For two $\operatorname{arcs} a$ and $b$, we write $a \rightsquigarrow b$ if there is a nice $t$-arc with starting arc $a$ and ending $\operatorname{arc} b$. This relation is transitive and is also preserved by $G$; hence, by Lemma 2.2, it is an equivalence relation. Let $a=(x, z)$ and let $E=[a]_{\rightsquigarrow}$ be the equivalence class of $a$ under $\rightsquigarrow$. We will show that every arc of $\Gamma$ is in $E$.

Let $(u, v) \in E$. By arc-transitivity, there exists a nice 2-arc $(u, v, w)$. Clearly, $(v, w) \in E$ and then, because $\rightsquigarrow$ is an equivalence relation preserved by $G$, the class $E$ is preserved setwise by both $G_{u v}$ and $G_{v w}$ and hence by $G_{v}$. In particular, all arcs incident to $v$ are in $E$. Repeating this argument using transitivity of $G$ and connectedness of $\Gamma$ allows us to conclude that every arc of $\Gamma$ is in $E$.

Now, let $g \in G_{0}$ and let $A$ be a nice $s$-arc stabilized by $g$. If $g$ acts transitively on the $p$ nice successors of $A$ then $G$ acts transitively on nice $(s+1)$-arcs, which is a contradiction. Since $L_{x}^{Y} \cong \mathbb{Z}_{p}$, it follows that $g$ stabilizes each nice successor of $A$. As we showed above, every arc of $\Gamma$ is in $\left[\left(v_{s-1}, v_{s}\right)\right]_{\rightsquigarrow}$ and therefore $g=1$.

Our next goal is to bound $s$. First, we need the following result.

Lemma 2.4 [6, Lemma 1]. Let $x$ and $g$ be elements of $G$. Put $x_{i}=x^{g_{i}}$ for $i \in \mathbb{Z}$ and define $H_{i}=\left\langle x_{1}, \ldots, x_{i}\right\rangle$ for each $i \geq 1$. Let $H_{0}=1$. Suppose that $x$ has prime order $p$ and that there exist positive integers $t$ and $n$ such that:

(1) $\left\langle H_{t}, g\right\rangle=G$;

(2) $\left|H_{i}: H_{i-1}\right|=p$, for $1 \leq i \leq t$; and

(3) $H_{t}$ contains no nonidentity normal subgroup of $G$ and no nonidentity subgroup of the center of $H_{t+n}$.

Then $t \leq 3 n$ and $t \neq 3 n-1$. Moreover, if $n=2, p=2$, and $t=6$, then $H_{t}$ contains $a$ nonidentity normal subgroup of $H_{8}$. 
The lemma as stated above is due to Glauberman but the proof is essentially due to Sims [11, Proposition 2.6]. It draws heavily on ideas contained in two papers of Tutte $[16,17]$. We are now ready to prove our main result.

Proof of TheOREM 1.2. Let $(\Gamma, G)$ be a locally $p$-sub-regular pair. We need to show that the order of $G_{u v}$ is equal to $p^{s-1}$ where $s \leq 7, s \neq 6$, and that, if $p=2$, then $s \leq 5$. Let $z$ be a vertex of $\Gamma$ and let $L=G_{z}^{\Gamma(z)}$. Since $(\Gamma, G)$ is a locally $p$-sub-regular pair, there exist neighbors $x, y \in \Gamma(z)$ such that $L_{x}^{Y} \cong \mathbb{Z}_{p}$ and $L=L^{\circ}$, where $Y=y^{L_{x}}$ and $L^{\circ}=\left\langle L_{x}, L_{y}\right\rangle$. Since $L=L^{\circ}$ and $L$ is transitive, $x^{L^{\circ}}=\Gamma(z)$. This shows that the hypothesis of Lemma 2.3 is satisfied.

Let $s$ be the largest integer such that $G$ acts transitively on $s$-arcs of $\Gamma$ that are nice with respect to $(x, z, y)$ and $G$. Let $\left(v_{0}, \ldots, v_{s}\right)$ be a nice $s$-arc of $\Gamma$ and let $G_{i}$ be the pointwise stabilizer of $\left(v_{0}, \ldots, v_{s-i}\right)$. By Lemma 2.3, we know that $\left|G_{i}\right|=p^{i}$ for $0 \leq i \leq s-1$. In particular, $\left|G_{1}\right|=p$. Let $x_{0}$ be a generator of $G_{1}$, let $g \in G$ be such that $\left(v_{1}, \ldots, v_{s}\right)^{g}=\left(v_{0}, \ldots, v_{s-1}\right)$ and let $x_{i}=x_{0}^{g^{i}}$. Finally, let $H_{i}=\left\langle x_{0}, \ldots, x_{i-1}\right\rangle$ and let $H_{0}=1$. The rest of the proof is split into three steps.

STEP 1 . We will show that $G_{i}=H_{i}$ for $0 \leq i \leq s$ by induction on $i$. It is clearly true for $i=0$. Let $1 \leq i \leq s$ and suppose that the statement is true for $i-1$. This implies that $H_{i}=\left\langle H_{i-1}, x_{i-1}\right\rangle=\left\langle G_{i-1}, x_{i-1}\right\rangle$. It is easy to check that $\left\langle G_{i-1}, x_{i-1}\right\rangle \subseteq G_{i}$. Note that $x_{0}$ does not fix $v_{s}$, hence $x_{i-1}=x_{0}^{g^{i-1}}$ does not fix $v_{s}^{g^{i-1}}=v_{s-i+1}$, therefore $x_{i-1} \notin G_{i-1}$. If $i<s$, we have $\left|G_{i}: G_{i-1}\right|=p$ and hence $G_{i}=\left\langle G_{i-1}, x_{i-1}\right\rangle=H_{i}$. If $i=s$, let $v_{-1}=v_{0}^{g}$. Clearly, $\left(v_{-1}, v_{0}, v_{1}\right)$ is a nice 2-arc. Note that, by the Frattini argument, the fact that $L^{\circ}=L$ implies that $G_{v_{0}}=\left\langle G_{v_{0} v_{1}}, G_{v_{-1} v_{0}}\right\rangle$. It follows that

$$
G_{s}=G_{v_{0}}=\left\langle G_{v_{0} v_{1}}, G_{v_{-1} v_{0}}\right\rangle=\left\langle G_{s-1},\left(G_{s-1}\right)^{g}\right\rangle=\left\langle H_{s-1},\left(H_{s-1}\right)^{g}\right\rangle=H_{s} .
$$

This completes Step 1.

STEP 2. We will show that $H_{s+1}$ acts transitively on edges of $\Gamma$ and that $G=\left\langle H_{s+1}, g\right\rangle$. By Step 1, we know that $H_{s+1}=\left\langle H_{S},\left(H_{S}\right)^{g}\right\rangle=\left\langle G_{S},\left(G_{S}\right)^{g}\right\rangle=$ $\left\langle G_{v_{0}}, G_{v_{-1}}\right\rangle$. Since $\Gamma$ is connected and $G$-arc-transitive, this shows that $H_{s+1}$ acts transitively on the edges of $\Gamma$. Moreover, since $v_{-1}=v_{0}^{g}$, this also shows that $G=\left\langle H_{S+1}, g\right\rangle$. This completes Step 2.

STEP 3. Note that $G$ acts transitively on edges of $\Gamma$ and, as was shown in Step 2, so does $H_{s+1}$. Since $H_{s-1}=G_{v_{0} v_{1}}$ is a $G$-arc-stabilizer, it follows that $H_{s-1}$ contains no nonidentity normal subgroup of $G$ and no nonidentity normal subgroup of $H_{s+1}$. This allows us to apply Lemma 2.4 (with $n=2$ and $t=s-1$ ) to conclude that $s \leq 7$ and $s \neq 6$ and, if $p=2$, then $s \leq 5$. This concludes the proof of Theorem 1.2.

\section{3. $p$-sub-regular actions}

We will now study $p$-sub-regular actions in more detail. We first recall the definition. Let $p$ be a prime. We say that a transitive action of a group $L$ on a 
set $\Omega$ is $p$-sub-regular if there exist $x, y \in \Omega$ such that $L=\left\langle L_{x}, L_{y}\right\rangle$ (Condition 1) and $L_{x}^{Y} \cong \mathbb{Z}_{p}$, where $Y=y^{L_{x}}$ (Condition 2). We now need a few more definitions which will be needed to characterize $p$-sub-regular actions.

Definition 3.1. Let $\Gamma=(V, A)$ be a digraph. For $n$ an even natural number, an alternating $n$-arc $U=\left(u_{0}, \ldots, u_{n}\right)$ is a sequence of $n+1$ vertices of $\Gamma$ such that, for every even integer $i$ with $0 \leq i \leq n-2$, both $\left(u_{i}, u_{i+1}\right)$ and $\left(u_{i+2}, u_{i+1}\right)$ are in $A$. $\Gamma$ will be called alternating-connected if, for any pair of vertices $x, y \in V$, there is an alternating $n$-arc with start-vertex $x$ and end-vertex $y$.

It is worth mentioning that alternating-connected digraphs are precisely the altercomplete digraphs of alter-exponent 1 , as defined in [8]. We now characterize $p$-subregular actions as groups of automorphisms of certain digraphs.

THEOREM 3.2. Let $p$ be a prime and let $L$ be a group acting on a set $\Omega$. Then this action is p-sub-regular if and only if there exists an L-arc-regular and alternatingconnected digraph $\Gamma=(\Omega, A)$ of out-valency $p$.

ProOF. $(\Leftarrow)$ Let $\Gamma=(\Omega, A)$ be an $L$-arc-regular and alternating-connected digraph of out-valency $p$. Clearly, $L$ is transitive on $\Omega$. Let $(x, y)$ be an $\operatorname{arc}$ of $\Gamma$. It is not hard to see that the set of end-vertices of alternating $n$-arcs with start-vertex $x$ is closed under the natural action of $\left\langle L_{x}, L_{y}\right\rangle$. Since $\Gamma$ is alternating-connected, this implies that $\left\langle L_{x}, L_{y}\right\rangle$ acts transitively on $\Omega$, and Condition 1 follows. Condition 2 follows from the assumption that $\Gamma$ is $L$-arc-regular and has out-valency $p$.

$(\Rightarrow)$ Let $L$ act $p$-sub-regularly as witnessed by $x, y \in \Omega$, and let $\Gamma=\left(\Omega,(x, y)^{L}\right)$. By definition, $\Gamma$ is $L$-arc-transitive. By Condition $2, \Gamma$ has out-valency $p$. Consider the set $X$ of end-vertices of alternating $n$-arcs with start-vertex $x$. Clearly, $x \in X$, and $X$ is closed under $L_{x}$. If $\left(x, x_{1}, \ldots, x_{n}\right)$ is an alternating $n$-arc and $g \in L_{y}$, then $\left(x, y, x^{g},\left(x_{1}\right)^{g}, \ldots,\left(x_{n}\right)^{g}\right)$ is an alternating $(n+2)$-arc, and hence $X$ is closed under $L_{y}$. By Condition $1, X$ is closed under $L$. Since $L$ is transitive on $\Omega$, this implies that $X=\Omega$ and $\Gamma$ is alternating-connected. Since $\Gamma$ is $L$-arc-transitive, this implies that $\Gamma$ is connected.

We now show that $L_{x y}=1$. Note that $\Gamma$ is connected hence there exists a sequence $\left(V_{0}, V_{1}, \ldots, V_{n}\right)$ of subsets of $\Omega$ such that $V_{0}=\{x\}, V_{n}=\Omega$ and $V_{i+1}=V_{i} \cup \Gamma^{+}\left(v_{i}\right)$ for some $v_{i} \in V_{i}$. Let $L_{i}$ be the pointwise stabilizer of $V_{i}$. By Condition 2, $\left|L_{i}: L_{i+1}\right|$ divides $p$. Since $L_{n}=1$, it follows that $L_{0}=L_{x}$ is a $p$-group. Moreover, we know that $\left|L_{x y}\right|=\left|L_{x y}^{\Gamma^{-}(y)}\right|\left|\bigcap_{v \in \Gamma^{-}(y)} L_{v y}\right|$ and, since $p$ does not divide $\left|L_{x y}^{\Gamma^{-}(y)}\right|$, we conclude that $L_{x y}^{\Gamma^{-}(y)}=1$. This, together with Condition 2, implies that $L_{x y}$ fixes $X$ pointwise. Since $X=\Omega$ we conclude that $L_{x y}=1$.

Hence, if $L$ acts $p$-sub-regularly on $\Omega$ as witnessed by $x, y \in \Omega$, then $L_{x y}=1$. This is the motivation for calling such actions $p$-sub-regular. It also suggests that $p$-subregular actions are, in some sense, close to being Frobenius. The following examples will show that many $p$-sub-regular actions of small degree are in fact Frobenius. 
ExAmples. Our first four infinite families of examples are all Frobenius groups. Hence, they can be represented as semidirect products $G=K \rtimes H$ where the action under consideration is that of $G$ on $K$ where $K$ acts regularly by multiplication and $H$ acts by conjugation.

(1) $\mathbb{Z}_{n} \rtimes \mathbb{Z}_{2}=\left\langle a, b \mid a^{2}=b^{n}=(a b)^{2}=1\right\rangle \cong \mathrm{D}_{n}$, for $n \geq 3$ odd. These are dihedral groups. Here, $p=2$.

(2) $\mathbb{Z}_{q} \rtimes \mathbb{Z}_{p}=\left\langle a, b \mid a^{p}=b^{q}=a^{-1} b a b^{m}=1\right\rangle$, for odd primes $p$ and $q$ with $p \mid(q-1)$ and $m$ an element of order $p$ in $\mathbb{Z}_{q}^{*}$. These are subgroups of affine transformations of the field of order $q$.

(3) $\left(\mathbb{Z}_{2}\right)^{n} \rtimes \mathbb{Z}_{p}$, for $n \geq 2$ and $p=2^{n}-1$ a prime. This is the group of affine transformations of the field of order $2^{n}$.

(4) $\left(\mathbb{Z}_{n}\right)^{p-1} \rtimes \mathbb{Z}_{p}=\left\langle b_{1}, \ldots, b_{p-1}\right\rangle \rtimes\langle a\rangle$ where $\left(b_{i}\right)^{a}=b_{i+1}$ for $1 \leq i \leq p-2$ and $\left(b_{p-1}\right)^{a}=\left(b_{1} \cdots b_{p-1}\right)^{-1}$, for $p$ an odd prime and $n \geq 2$ not divisible by 3 .

The next two infinite families are closely related to each other.

(1) $S L(2, p)$ acting on the $p^{2}-1$ nonzero vectors in $\left(\mathbb{Z}_{p}\right)^{2}$, for $p$ an odd prime. This is the usual action of matrices acting on vectors by multiplication.

(2) $\operatorname{PSL}(2, p)$ acting on $\left(p^{2}-1\right) / 2$ points, for $p$ an odd prime. Actions in this family are induced from actions in the previous family by identifying opposite pairs of matrices and opposite pairs of vectors.

For $p>3$, the groups in the above two families are not Frobenius. There are also some nice examples of small degree that do not fall in any of the above families, such as $A_{5}$ acting on the 20 vertices of the dodecahedron. This action is obviously not Frobenius. It is also imprimitive, as opposite vertices of the dodecahedron form a block system.

An exhaustive computer search through a library of transitive group actions of small degree reveals that, apart from $\mathrm{A}_{5}$ acting on the vertices of the dodecahedron, all $p$ sub-regular actions of degree at most 26 appear in at least one of the six infinite families listed above. Some of theses actions are primitive and hence part of our work can be viewed as an effort towards the Weiss conjecture. On the other hand, the imprimitive actions also need to be considered in view of the more general Problem 1.3. From the perspective of this problem, most of the past efforts have been concentrating on the locally primitive graphs, thus working in the setting of the Weiss conjecture. We hope that our results will spur further investigation of arc-stabilizers in locally imprimitive graphs, and perhaps shed new light on the problem of bounding the order of arcstabilizers in arc-transitive graphs in general.

\section{Acknowledgement}

This work was done under the supervision of Primož Potočnik at the University of Ljubljana. 


\section{References}

[1] M. D. E. Conder and P. Dobcsányi, 'Trivalent symmetric graphs on up to 768 vertices', J. Combin. Math. Combin. Comput. 40 (2002), 41-63.

[2] M. D. E. Conder, C. H. Li and C. E. Praeger, 'On the Weiss conjecture for finite locally-primitive graphs', Proc. Edinb. Math. Soc. (2) 43 (2000), 129-138.

[3] M. D. E. Conder, A. Malnič, D. Marušič and P. Potočnik, 'A census of semisymmetric cubic graphs on up to 768 vertices', J. Algebraic Combin. 23 (2006), 255-294.

[4] A. Gardiner, 'Arc transitivity in graphs', Q. J. Math. 24 (1973), 399-407.

[5] _ 'Doubly primitive vertex stabilisers in graphs', Math. Z. 135 (1974), 257-266.

[6] G. Glauberman, 'Normalizers of $p$-subgroups in finite groups', Pacific J. Math. 29 (1969), 137-144.

[7] M. A. Iranmanesh, 'On finite $G$-locally primitive graphs and the Weiss conjecture', Bull. Aust. Math. Soc. 70 (2004), 353-356.

[8] D. Marušič and P. Potočnik, 'Bridging semisymmetric and half-arc-transitive actions on graphs', European J. Combin. 23 (2002), 719-732.

[9] P. Potočnik, 'A list of 4-valent 2-arc-transitive graphs and finite faithful amalgams of index $(4,2)$ ', European J. Combin. 30 (2009), 1323-1336.

[10] A. Q. Sami, 'Locally dihedral amalgams of odd type', J. Algebra 298 (2006), 630-644.

[11] C. C. Sims, 'Graphs and finite permutation groups', Math. Z. 95 (1967), 76-86.

[12] V. I. Trofimov, 'Stabilizers of the vertices of graphs with projective suborbits', Soviet Math. Dokl. 42 (1991), 825-828.

[13] _ _ 'Graphs with projective suborbits', Izv. Akad. Nauk SSSR Ser. Mat. 55 (1991), 890-916 (in Russian).

[14] - 'Vertex stabilizers of locally projective groups of automorphisms of graphs. A summary', in: Groups, Combinatorics and Geometry, Durham (World Scientific, River Edge, NJ, 2001), pp. 313-326.

[15] V. I. Trofimov and R. Weiss, 'Graphs with a locally linear group of automorphisms', Math. Proc. Cambridge Philos. Soc. 118 (1995), 191-206.

[16] W. T. Tutte, 'A family of cubical graphs', Proc. Cambridge Philos. Soc. 43 (1947), 459-474.

[17] , 'On the symmetry of cubic graphs', Canad. J. Math. 11 (1959), 621-624.

[18] R. Weiss, 's-transitive graphs', Colloq. Math. Soc. János Bolyai 25 (1978), 827-847.

[19] H. Wielandt, Finite Permutation Groups (Academic Press, New York, 1964).

GABRIEL VERRET, Institute of Mathematics, Physics and Mechanics, Jadranska 19, 1000 Ljubljana, Slovenia

e-mail: gabriel.verret@fmf.uni-lj.si 